\title{
1 Vpr Co-assembles with Gag during HIV-1 Assembly
}

2 Kate Bredbenner ${ }^{1}$ and Sanford M Simon ${ }^{1 *}$

3

4

5 1. Lab of Cellular Biophysics, Rockefeller University, 1230 York Ave, New York, NY 10065

$6 \quad{ }^{*}$ Corresponding Author: Simon@rockefeller.edu 


\begin{abstract}
The HIV-1 accessory protein Vpr is packaged into new virions at a 7:1 ratio of Gag/Vpr. Previous biochemical and genetic analysis has shown that Vpr gets packaged into virions via an LXXLF motif on the p6 domain of the Gag structural polyprotein. The kinetics of Vpr packaging compared to Gag assembly was previously unknown. Here, we confirm via biochemistry and imaging that fluorescently tagged Vpr gets packaged into virus-like particles only when the LXXLF motif is intact. When the LXXLF motif is mutated, Vpr is no longer recruited to Gag assemblies. When Vpr and Gag assembly are imaged together, we see that Vpr co-assembles with a slight delay compared to Gag suggesting that $\mathrm{Vpr}$ is not being recruited to the membrane with Gag but is instead being recruited to actively assembling Gag.
\end{abstract}

\title{
Importance
}

HIV-1 affects over 30 million people around the globe, and although we have good treatments, there is still no cure. The virus encodes 15 distinct proteins, and four of those proteins are known as accessory proteins. $\mathrm{Vpr}$ is one of the accessory proteins that is packaged into HIV-1 by interacting with the Gag structural protein. Without Vpr, HIV-1 is not as infectious. Our research shows that $\mathrm{Vpr}$ is packaged into new viruses as the virus is being formed rather than being put in towards the end of the assembly of a virus. Getting a clearer view of each step in the process of assembling each virion will help inform future treatments and help with overall comparisons between the assembly of different viruses.

\section{Introduction}

HIV-1 viruses assemble on the plasma membrane of infected cells via polymerization of the p55Gag structural polyprotein (1). It takes between 5-25 minutes between the arrival of the HIV-1 genome and assembly of the Gag protein $(2,3)$. Each virus contains approximately $2400 \mathrm{Gag}$ polyproteins (4). After recruitment of Gag is complete, host-cell ESCRT proteins are recruited transiently to the sites of assembly in order to help the particle scission from the cell (5-8). Within the p6 domain of Gag, the PTAP motif recruits TSG101 $(9,10)$ and the YPXL motif recruits Alix (11-14) which are both necessary for viral budding and release $(9,12,15)$. Alix also helps recruit other downstream ESCRT components necessary for scission such as CHMP4B and VPS4A $(8,11,13,14)$.

HIV-1 also encodes several other proteins that are packaged into virions. The Pol proteins (protease, reverse transcriptase, and integrase) are synthesized and packaged via a fusion to Gag. They are made as a consequence of a -1 ribosomal frameshift that occurs approximately $5 \%$ of the time resulting in approximately 120 copies of the Pol proteins per virion(16). HIV-1 also encodes several additional accessory proteins such as Vif, Nef, and $\mathrm{Vpr}$ that are packaged into virions.

Vpr is a 97-amino acid accessory protein that is packaged into virions via interaction with the LXXLF motif located in the p6 domain of Gag $(17,18)$. The LXXLF motif on p6 is further towards the carboxy terminus than both the PTAP and the YPXL motifs which recruit the ESCRT machinery. Vpr is packaged into each virus with a ratio of Gag/Vpr of 7:1, which corresponds to approximately 340 molecules per virion (19-22). Although $\mathrm{Vpr}$ is not 
necessary for HIV-1 growth in culture, it might play a role during in vivo replication $(23,24)$. Proposed functions of $\mathrm{Vpr}$ include influences on nuclear entry of viral proteins $(25,26)$ and induction of G2/M cell cycle arrest or cell apoptosis (27-29).

Because of its ability to interact with Gag and be packaged in-trans, Vpr has been a popular target to use for fusions to deliver proteins into viruses. Previous research has used Vpr to deliver the HIV-1 protease, integrase, and reverse transcriptase proteins into virions outside of their typical packing as fusions to Gag (30-34). Other research has packaged the TEV protease or GFP into virions with $\mathrm{Vpr}$ (35-39).

The kinetics of the recruitment of $\mathrm{Vpr}$ and packaging into viruses is an open question. To determine the kinetics, we fused an mEGFP to Vpr and confirmed that it was packaged into virus-like particles (VLPs). We also confirmed that a premature truncation of the Gag polyprotein, resulting in a partial truncation of the LXXLF motif, prevented packaging of $\mathrm{Vpr}$, but still allowed assembly and release of VLPs. The recruitment of Vpr during assembly of VLPs shows that it recruits with a slight delay compared to Gag.

\section{Results}

\section{Vpr is incorporated into VLPs}

To determine the kinetics of $\mathrm{Vpr}$ recruitment, we wanted to be able to image $\mathrm{Vpr}$ using fluorescence. Thus, we first had to confirm that fluorescently tagged Vpr was packaged specifically into our VLPs. We created an mEGFP-Vpr construct and co-expressed it with an NL4.3-GagPol packaging vector with an mCherry inserted after the matrix domain of Gag (GagPol-mCherry) (Fig 1). This latter construct will make mostly Gag-mCherry, but also GagPol-mCherry when slippage occurs. The expression and packing of the mEGFP$\mathrm{Vpr}$ into VLPs was confirmed by Western blots (Fig 2A). To confirm that Vpr was specifically being recruited to sites of assembly via the LXXLF motif, we introduced a stop codon into the LXXLF motif of Gag which removed the last 9AA of p6 while leaving the Alix and TSG101 recruitment motifs intact (GagPol-mCherry-LXXLF) (Fig 1). This mutation also leaves the Pol amino acid sequence fully intact. When we co-expressed mEGFP-Vpr with GagPol-mCherry-LXXLF, we were able to collect VLPs that that were positive for capsid and mCherry, albeit at reduced levels, but mEGFP-Vpr was not detectable (Fig 2A).
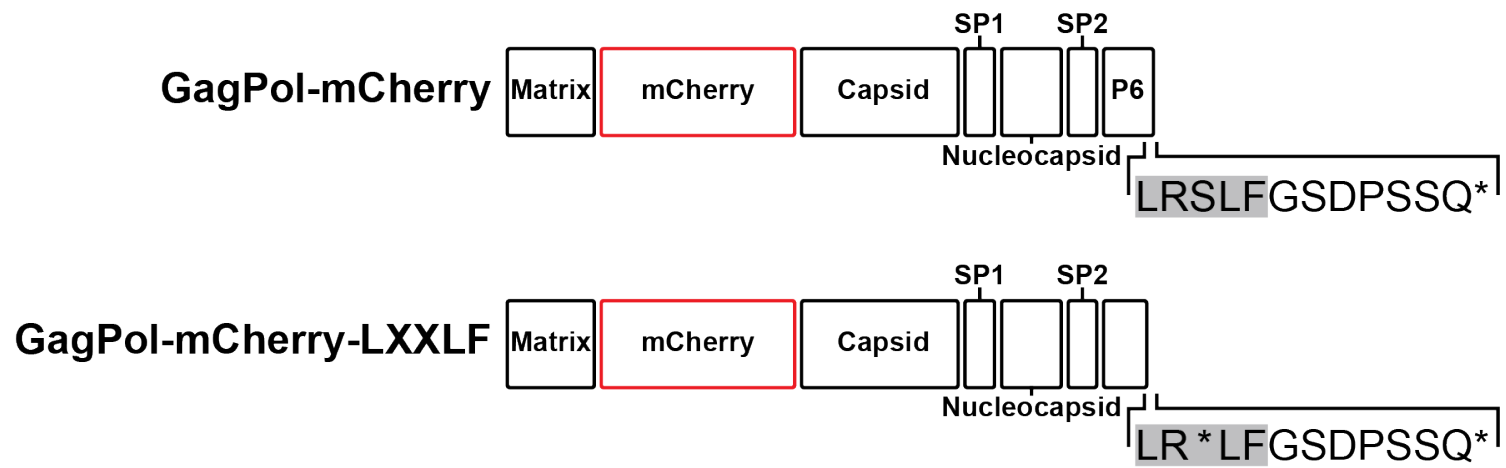

Figure 1 Schematic of GagPol Plasmids 
The sequences for GagPol-mCherry and GagPol-mCherry-LXXLF were constructed from NL4.3 sequences which have the slippage site intact. The $m$ Cherry is inserted in each construct, without a linker, at the end of matrix domain prior to the Matrix/Capsid cleavage site. GagPol-mCherry-LXXLF has a stop codon introduced in the middle of the LXXLF Vpr recruitment motif which removes the last 9 amino acids from Gag but leaves the Pol coding sequence intact.

To further test the assembly of mEGFP-Vpr into VLPs, we co-transfected it together with GagPol-mCherry and collected supernatant after 24 hours. There are some potential problems with this approach. First, VLPs are created via transient transfection, which means that the VLPs come from a population of cells with different amounts of plasmid and expressing differing levels of each protein. Further, the collection of VLPs includes small pieces of cell debris that go through our filter. For this reason, quantifying the relative levels of proteins VLPs collected from a mixed population of cells has potential limitations for quantifying the packaging of Vpr. Additionally, VLPs with GagPol-mCherry can be excited at $488 \mathrm{~nm}$. While they will not be as efficiency excited as a GFP, at a high concentration VLPs with GagPol-mCherry excited at $488 \mathrm{~nm}$ may falsely be recorded as positives for mEGFP-Vpr. To limit any false positives, we imaged VLPs $(n=100)$ with GagPol-mCherry and GagPol-mCherry-LXXLF in the absence mEGFP-Vpr to quantify the cross-channel contamination so the mCherry excited by the $488 \mathrm{~nm}$ laser can be subtracted from the mEGFP images.

Despite the stringent subtraction of the mCherry signal from the GFP channel and the inherent problems with collecting VLPs from transient transfection, $51 \%$ of all GagPolmCherry VLPs also contained detectable mEGFP-Vpr (Fig 2B). When we analyzed VLPs made with GagPol-mCherry-LXXLF which is missing the Vpr recruitment domain, the percent of GagPol-mCherry VLPs which contained detectable mEGFP-Vpr dropped to $9 \%$ (Fig 2B).

To test if mEGFP-Vpr was only found in VLPs and not found in either other cell debris or in puncta without Gag, we checked mEGFP-Vpr positive puncta $(n=100)$ and found that $77 \%$ of them were also GagPol-mCherry positive (Fig 2C). When we transfect cells with GagPol encoding an embedded fluorescent protein, we co-transfect with a plasmid with GagPol without a tag. This avoids morphological problems in VLPs that occur when all of the copies of GagPol are expressed as fusion proteins (40). The ratio we use is a 1:4 ratio of tagged/untagged GagPol. We believe that the percentage of mEGFP-Vpr puncta that also contain GagPol-mCherry is not $100 \%$ due to a proportion of cells that were only transfected with untagged GagPol and mEGFP-Vpr without tagged GagPol. The results from Western blots and imaging are consistent with mEGFP-Vpr being packaged specifically into VLPs. We also confirm that the LXXLF motif is necessary for that recruitment. 

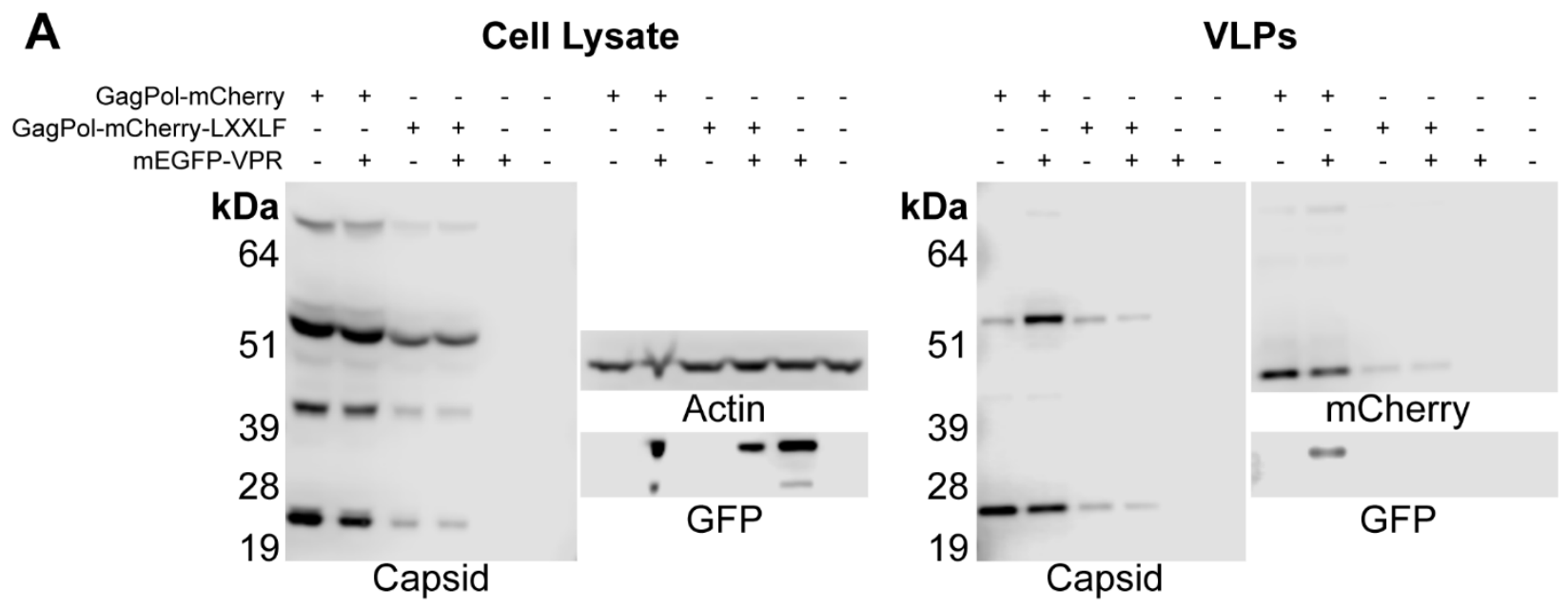

B

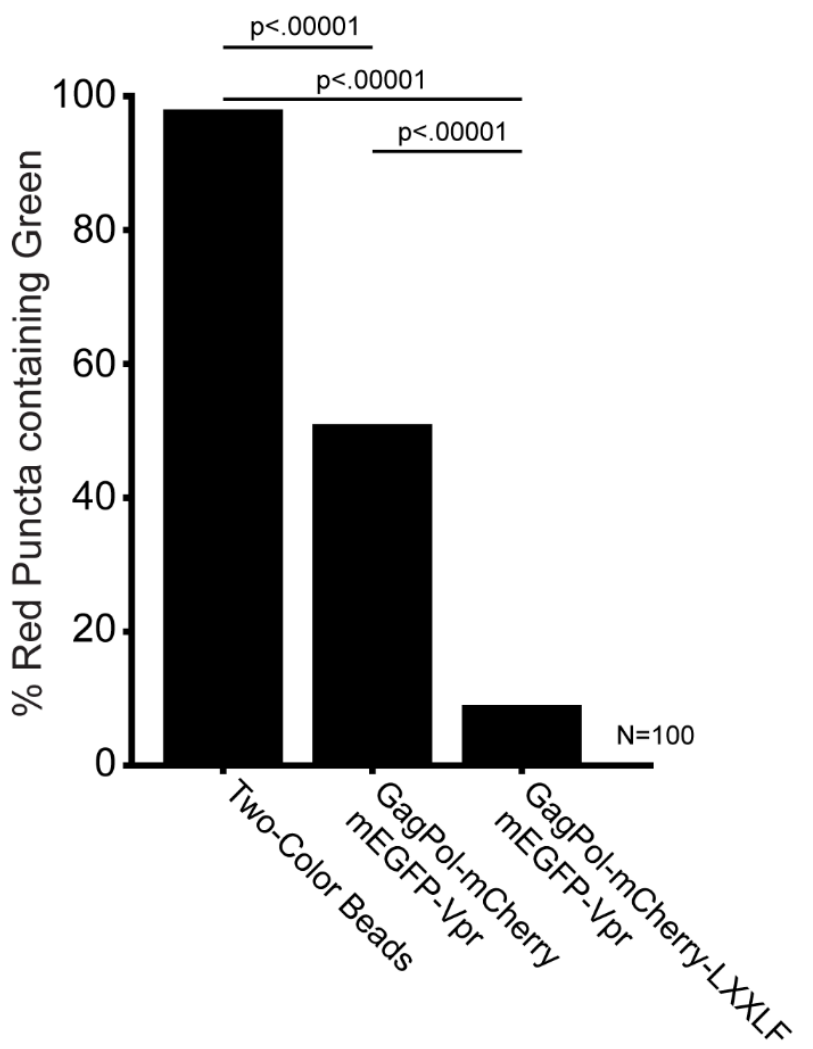

C

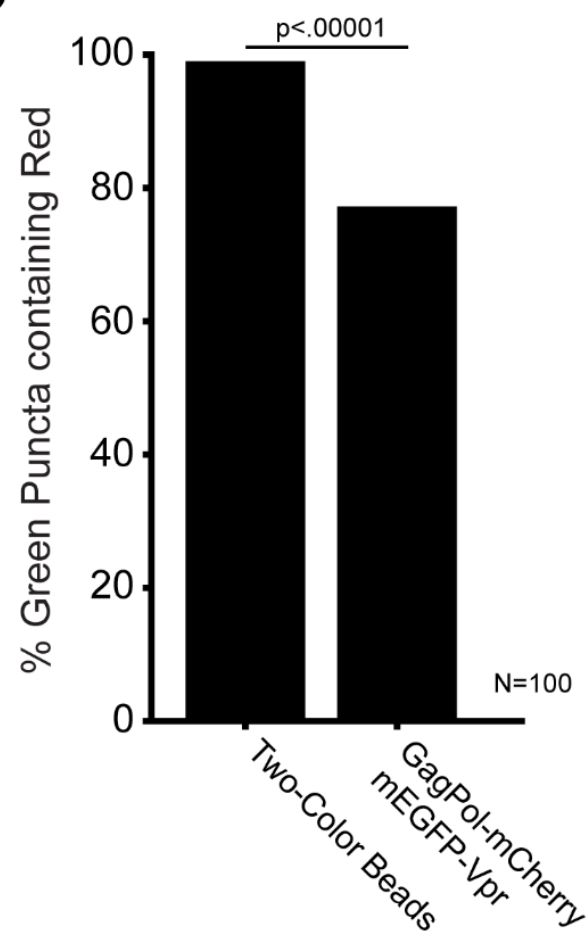

Figure 2 Vpr is packaged specifically into VLPs

A Cell lysate (left) and VLPs (right) collected from Hek293T cells 48 hours after transfection. Cell lysate was probed with anti-capsid, anti-actin, and anti-GFP antibodies. Western blots were probed with anti-capsid, anti-mCherry, and antiGFP antibodies. B shows the quantification of the percent of red puncta that were also green $(n=100)$. C shows the quantification of the percent of green puncta that were also red $(n=100)$. 


\section{Vpr co-assembles with GagPol}

142 We next examined the recruitment of mEGFP-Vpr in cells expressing either GagPol143 mCherry or GagPol-mCherry-LXXLF. Still images from cells with GagPol-mCherry and 144 mEGFP-Vpr six hours after transfection showed puncta in both red and green that co145 aligned. In contrast, when we co-transfected the GagPol-mCherry-LXXLF with the 146 mEGFP-Vpr, cells assembled VLPs, but mEGFP-Vpr was not detected at sites of GagPol 147 (Fig 3A). Quantification of VLPs in the still images showed that $78 \%$ of the selected 148 GagPol-mCherry puncta on the cell were also mEGFP-Vpr positive, but only $5 \%$ of 149 GagPol-mCherry-LXXLF puncta were mEGFP-VPR positive (Fig 3B). The percentage of 150 GagPol-mCherry puncta that were positive for mEGFP-Vpr on the surface of cells was 151 higher than from isolated VLPs. This substantiates the conclusion that a contributing 152 factor to the lower level of colocalization in the VLPs were cells expressing GagPol153 mCherry but not mEGFP-Vpr. 


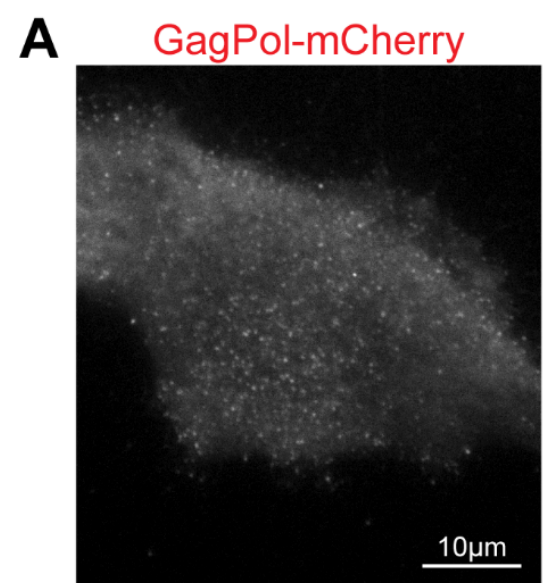

GagPol-mCherry-LXXLF

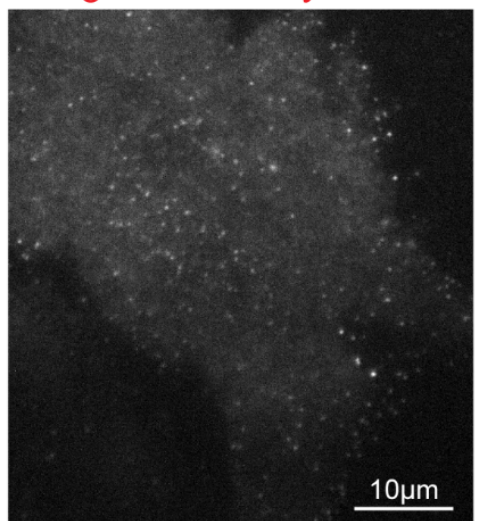

B

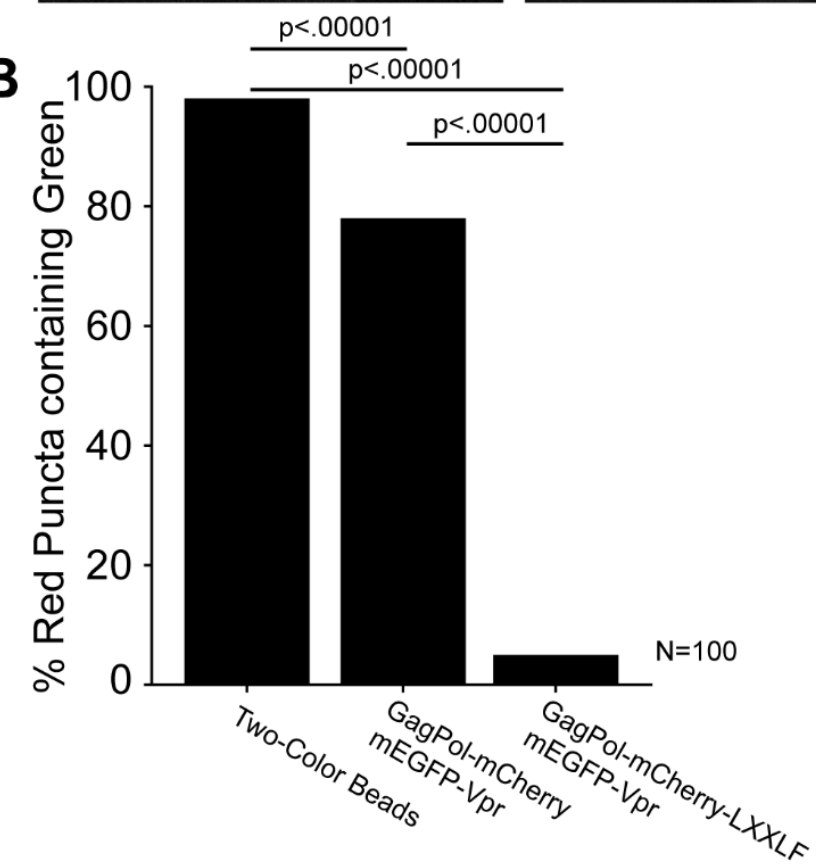

mEGFP-Vpr

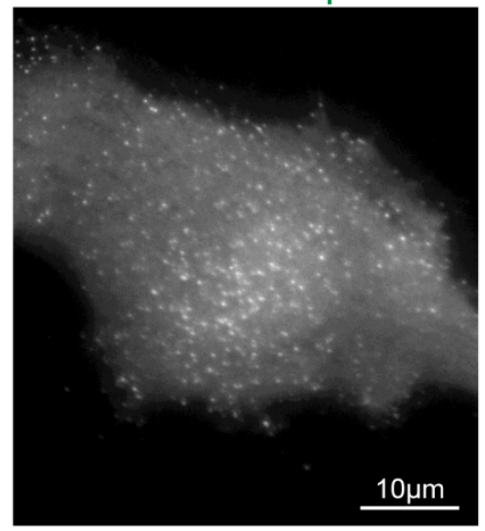

mEGFP-Vpr

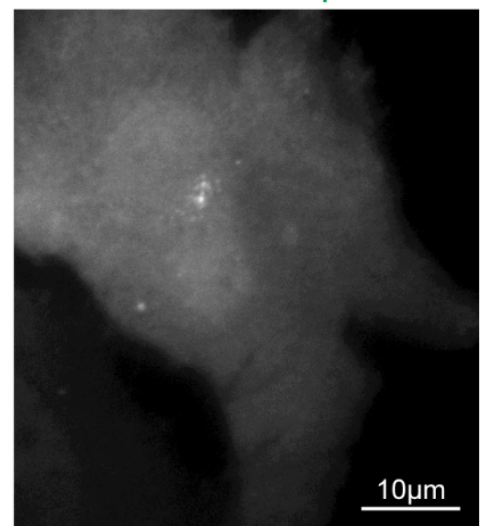

$10 \mu \mathrm{m}$
Merge

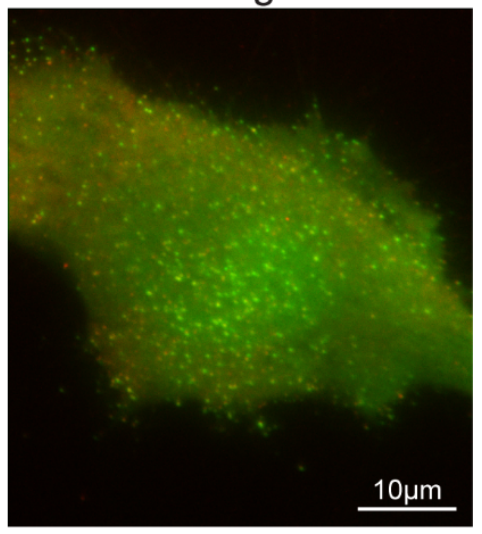

Merge

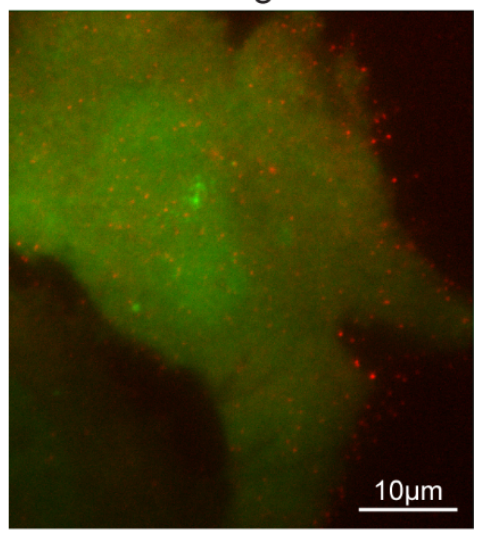


Single frames from a sequence of HeLa cells actively assembling new particles (A). The top row of images is from cells transiently expressing GagPol-mCherry and mEGFP-Vpr. The bottom row is from cells transiently expressing GagPol$m C h e r r y-L X X L F$ and mEGFP-Vpr. The first column is GagPol in red, the second is $V p r$ in green, and the final column is a merge of the first two. All scale bars are $10 \mu \mathrm{m}$. Quantification of puncta from two-color beads and GagPol-mCherry or GagPol-mCherry-LXXLF puncta on from cells $(n=100)$.

To determine the kinetics of recruitment of Vpr to VLPs, we imaged mEGFP-Vpr in live cells. When we did a time lapse imaging of mEGFP-Vpr together with GagPol-mCherry in HeLa cells, we saw that mEGFP-Vpr co-localized with GagPol (Fig 4A). The intensity of the fluorescence from mEGFP-Vpr reaches a plateau, indicating the end of net recruitment, similar to the plateau reached by GagPol assembly. Vpr does show a delay of 4-6 minutes in reaching its plateau when compared to GagPol. The kinetics or recruitment we observed of the mEGFP-Vpr was the same whether we used only untagged GagPol or a mixture with untagged and tagged GagPol-mCherry (Fig 4B). 
A

Two-Color Assemblies

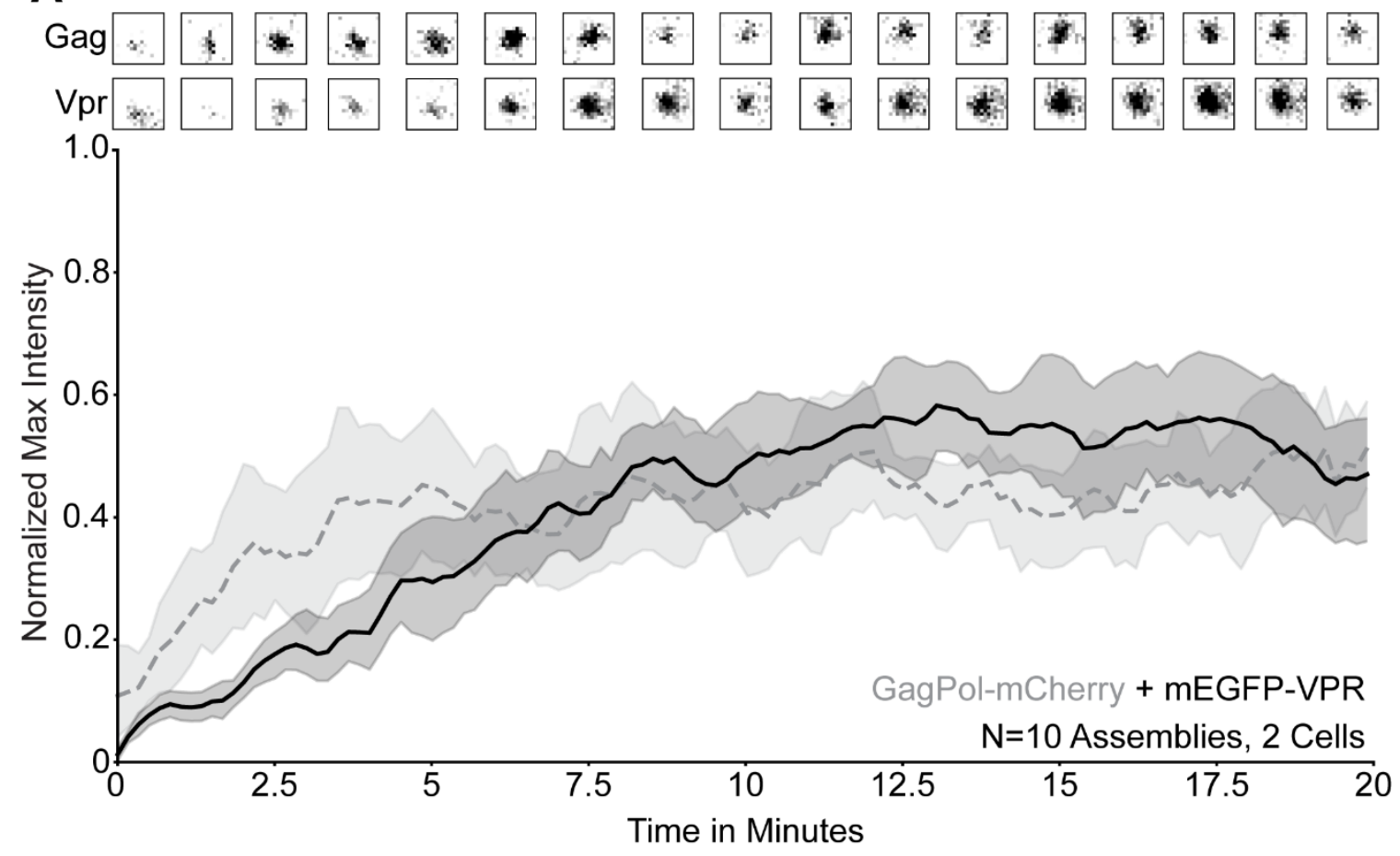

B Single Color Assemblies

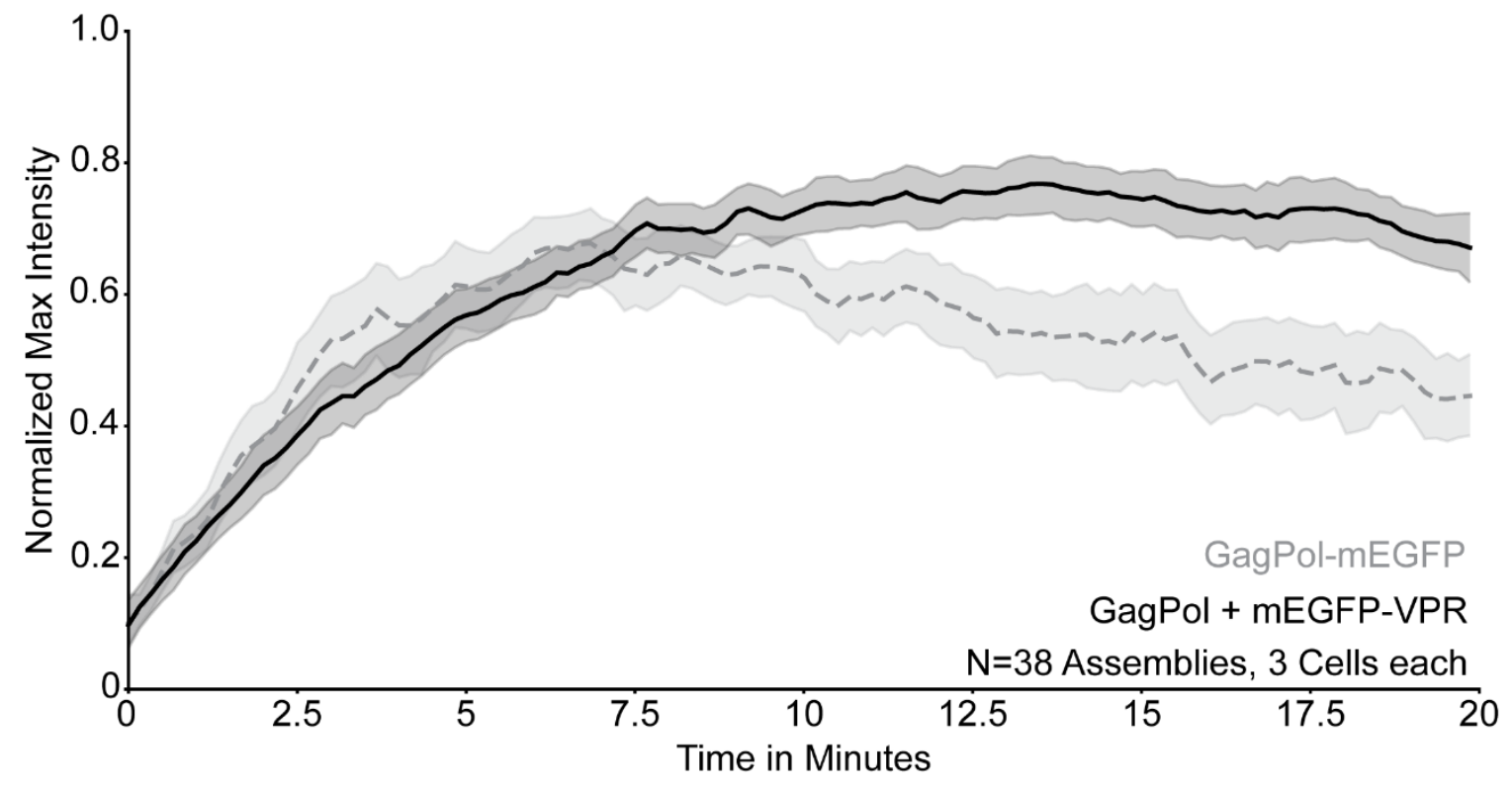

Figure 4 Assembly traces of GagPol and Vpr

A Assembly of Vpr and Gag-Pol. Top: select images from assembly. Bottom: The fluorescence intensity of the recruitment of mEGFP-Vpr and Gag-Pol-mCherry (average of 10 assemblies). B Traces for assembly traces for 38 VLPs where Vpr and GagPol were imaged separately in different cells. GagPol-mEGFP was imaged without Vpr. mEGFP-Vpr was imaged with untagged GagPol. The solid 
line in the graphs in both $\mathbf{A}$ and $\mathbf{B}$ shows the average while the shaded region surrounding the solid line shows the $95 \%$ confidence interval. Each assembly was rescaled to be on a $0-1$ axis via $\left(I-I_{\min }\right) /\left(I_{\max }-I_{\min }\right)$.

\section{Discussion}

Our data shows that $\mathrm{Vpr}$ co-assembles with Gag, but with a slight delay. We also confirm that the LXXLF motif is required for $\mathrm{Vpr}$ recruitment. The delay of assembly of $\mathrm{Vpr}$ compared to Gag suggests that Vpr might be recruited to Gag only after Gag is at the membrane rather than Vpr being attached to Gag before arriving at to the membrane. It has been shown previously that Vpr and Tsg101 can competitively bind to Gag since their recruitment sites are close together (41). The competition with Tsg101 may also contribute to the delay of $\mathrm{Vpr}$ assembly compared to Gag. Our imaging can detect overall fluorescence, but it cannot determine whether there is turnover at the LXXLF binding site. It is possible that Tsg101 and Vpr might influence each other when it comes to binding Gag. This potential interaction of Tsg101/Npr might also suggest why only $\sim 340 \mathrm{Vpr}$ proteins are found in VLPs when approximately $2400 \mathrm{Vpr}$ binding sites exist in a single assembled virus $(4,19)$.

Our findings also suggest that $\mathrm{Vpr}$ is a good way to monitor assembly in-trans without tagging or altering Gag. Any protein fused to Vpr would arrive early in the assembly process, stay through scission, and be present in the final VLP. This contrasts with proteins like the ESCRTs which only show up in the final stages of assembly and don't make it into the final VLP (5-8).

The incorporation kinetics of other accessory proteins like Vif and Nef are also unknown. It has been reported that Vif gets into particles by interactions with the viral RNA (42), and it is suspected that Nef gets into particles due to its myristoylation and interaction with membranes (16,43-46). Imaging could reveal other aspects of the kinetics of that packaging and give a fuller picture of how the HIV-1 virion is put together.

\section{Methods}

\section{Cell Culture}

HeLa and Hek293T cells were grown in Dulbecco's Modified Eagle's medium (DMEM, Gibco) supplemented with I-glutamine, sodium pyruvate, and $10 \%$ fetal bovine serum (FBS, Sigma). For live cell imaging HeLa cells were seeded onto MatTek dishes with no. 1.5 coverslips coated with fibronectin (Invitrogen). HeLa cells were transfected 6 hours before imaging with Fugene 6 (Promega) and 1000ng of DNA. Before imaging, media was replaced with cell imaging media (HBSS (Sigma), 10mM HEPES, pH7.4) supplemented with $1 \%$ FBS.

Hek293T cells were transfected with Lipofectamine 2000 (Thermo Fisher) and 1000ng of DNA 24 hours before VLP collection for imaging and 48 hours before VLP collection for westerns. 


\section{VLP Collection and Imaging}

Media from transfected Hek293T cells was collected either 24 or 48 hours after transfection and filtered through a $0.22 \mu \mathrm{m}$ filter (Millex). $1 \mathrm{~mL}$ of cell media was carefully pipetted over a $20 \%$ sucrose in PBS solution and centrifuged at $>30,000 \mathrm{~g}$ for 1 hour. All supernatant was removed and collected VLPs were either resuspended in 100uL PBS for imaging or in 65ul of RIPA Buffer for westerns.

The collected VLPs in PBS were placed onto Poly-D-Lysine coated MatTek dishes with a no. 1.5 coverslip. VLPs were allowed to adhere for 20 minutes in the dark, then $2.5 \mathrm{~mL}$ of PBS was added to each dish for imaging.

\section{Plasmids}

GagPol-mCherry, GagPol-mEGFP, GagPol-LXXLF, and GagPol-mCherry-LXXLF were all created from the PCRV1-NL4.3-GagPol packaging vector which was a gift from the Paul Bieniasz at Rockefeller University. Both mCherry and mEGFP were cloned with no linker after the Matrix domain in Gag via Gibson assembly with the HiFi DNA Assembly cloning kit (New England Biolabs). Similar constructs have been previously published. The LXXLF mutation inserts a premature stop codon at the 43 amino acid within p6 which removes the last 10 amino acids of $p 6$. This stop codon affects the Gag coding sequence, but makes no amino acid changes to the Pol reading frame. This mutation was created with the Quikchange site-directed mutagenesis kit (Agilent).

The mEGFP-Vpr construct was altered from the Addgene 110200 plasmid containing TEV-Vpr which was a gift from Sergi Padilla Parra (39). TEV was removed from the original plasmid and mEGFP was inserted in its place using a Gibson reaction via the HiFi DNA Assembly cloning kit (New England Biolabs).

\section{Biochemistry}

Hek293T cell lysates were collected by adding 200uL RIPA buffer directly to cells after removing media. Cells were kept on ice for 20 minutes before RIPA was collected, vortexed, and frozen overnight. Collected VLPs and cell lysate were run on a 4-12\% TrisGlycine gel (Novex). Either an Anti-HIV-1 p24 Monoclonal (183-H12-5C) obtained from the NIH AIDS Reagent Program $(47,48)$, a monoclonal anti-GFP (Living Colors JL-8, Clontech), or a monoclonal anti- $\beta$-actin (Sigma-Aldrich) primary antibody was used. HRPcoupled secondaries were used. Westerns were visualized on a LiCOR using ECL Prime.

\section{Assembly Selection and Plotting}

Videos of assembly of Gag or Vpr in HeLa cells were analyzed with Metamorph software. The camera has 100 units added to each pixel and this we subtracted from all frames. From there, individual assemblies were selected and trimmed to the first time the assembling puncta was visible and the last time the puncta was visible. This automatically sets the first frame of the assembly as time zero. To account for the differing intensities between mEGFP and mCherry and for the differing intensities between different cells, the 
$\frac{N-\min \left(I^{\text {Trace }}\right)}{\max \left(I^{\text {Trace }}\right)-\min \left(I^{\text {Trace }}\right)}$. After rescaling, a rolling filter was applied to the traces. The rescaled max intensity for each frame of the assemblies were plotted together using python.

\section{VLP Quantification}

VLPs from cells transfected only with GagPol-mCherry or GagPol-mCherry-LXXLF were collected and imaged with excitation at $488 \mathrm{~nm}$ and $594 \mathrm{~nm}(\mathrm{n}=100)$. Puncta present when imaged with $594 \mathrm{~nm}$ excitation were selected via with a $16 \times 16$ pixel circular region and the max intensities were recorded. The same regions were used to measure the max intensities during excitation at $488 \mathrm{~nm}$. The percentage of mCherry excitation at $488 \mathrm{~nm}$ was calculated to be $20.551 \%$ of the excitation at $594 \mathrm{~nm}$.

For VLPs from cells transfected with GagPol-mCherry and mEGFP-Vpr or GagPol$m$ Cherry-LXXLF and mEGFP-Vpr, puncta that were present when excited at 594nm were selected with circular regions and the max intensity was recorded. The same regions were then applied to an image taken with excitation at $488 \mathrm{~nm}$ and the max intensities were recorded. The max intensities from excitation at $488 \mathrm{~nm}$ had $20.551 \%$ of the max intensities with excitation at $594 \mathrm{~nm}$ subtracted from them. If the resulting value of max intensity with $488 \mathrm{~nm}$ excitation after subtraction was greater than zero, then that represented a red puncta that was also positive for green fluorescence.

\section{Statistics}

$P$ values for VLP quantification were calculated via a Chi-square test.

\section{Acknowledgements}

We would like to thank Daniel Scott Johnson at Hofstra University and Joan Pulupa at Rockefeller University for help building and maintaining our microscope. We would also like to acknowledge the National Institute of General Medical Sciences of the National Institutes of Health for an award to SMS: R01GM119585.

\section{References}

1. Jouvenet N, Neil SJD, Bess C, Johnson MC, Virgen CA, Simon SM, Bieniasz PD. 2006. Plasma membrane is the site of productive HIV-1 particle assembly. PLoS Biol 4:22962310. doi:10.1371/journal.pbio.0040435

2. Jouvenet N, Bieniasz PD, Simon SM. 2008. Imaging the biogenesis of individual HIV-1 virions in live cells. Nature 454:236-40. doi:10.1038/nature06998

3. Jouvenet N, Simon SM, Bieniasz PD. 2009. Imaging the interaction of HIV-1 genomes and Gag during assembly of individual viral particles. Proc Natl Acad Sci U S A 106:19114-9. doi:10.1073/pnas.0907364106

4. Carlson LA, Briggs JAG, Glass B, Riches JD, Simon MN, Johnson MC, Muller B, Grunewald K, Krausslich HG. 2008. Three-Dimensional Analysis of Budding Sites and 
Released Virus Suggests a Revised Model for HIV-1 Morphogenesis. Cell Host Microbe 4:592-599. doi:10.1016/j.chom.2008.10.013

5. Morita E, Sandrin V, McCullough J, Katsuyama A, Baci Hamilton I, Sundquist WI. 2011. ESCRT-III protein requirements for HIV-1 budding. Cell Host Microbe 9:235-242. doi:10.1016/j.chom.2011.02.004

6. Baumgärtel V, Ivanchenko S, Dupont A, Sergeev M, Wiseman PW, Kräusslich HG, Bräuchle C, Müller B, Lamb DC. 2011. Live-cell visualization of dynamics of HIV budding site interactions with an ESCRT component. Nat Cell Biol 13:469-476. doi:10.1038/ncb2215

7. Bleck M, Itano MS, Johnson DS, Thomas VK, North AJ, Bieniasz PD, Simon SM. 2014. Temporal and spatial organization of ESCRT protein recruitment during HIV-1 budding. Proc Natl Acad Sci U S A 111:12211-6. doi:10.1073/pnas.1321655111

8. Jouvenet N, Zhadina M, Bieniasz PD, Simon SM. 2011. Dynamics of ESCRT protein recruitment during retroviral assembly. Nat Cell Biol 13:394-401. doi:10.1038/ncb2207

9. Garrus JE, Von Schwedler UK, Pornillos OW, Morham SG, Zavitz KH, Wang HE, Wettstein DA, Stray KM, Côté M, Rich RL, et al. 2001. Tsg101 and the vacuolar protein sorting pathway are essential for HIV-1 budding. Cell 107:55-65. doi:10.1016/S00928674(01)00506-2

10. Martin-Serrano J, Zang T, Bieniasz PD. 2001. HIV-1 and Ebola virus encode small peptide motifs that recruit Tsg101 to sites of particle assembly to facilitate egress. Nat Med 7:1313-1319. doi:10.1038/nm1201-1313

11. Strack B, Calistri A, Craig S, Popova E, Göttlinger HG. 2003. AIP1/ALIX is a binding partner for HIV-1 p6 and EIAV p9 functioning in virus budding. Cell 114:689-699. doi:10.1016/S0092-8674(03)00653-6

12. Fisher RD, Chung HY, Zhai Q, Robinson H, Sundquist WI, Hill CP. 2007. Structural and Biochemical Studies of ALIXIAIP1 and Its Role in Retrovirus Budding. Cell 128:841-852. doi:10.1016/j.cell.2007.01.035

13. Von Schwedler UK, Stuchell M, Müller B, Ward DM, Chung HY, Morita E, Wang HE, Davis T, He GP, Cimbora DM, et al. 2003. The protein network of HIV budding. Cell 114:701-713. doi:10.1016/S0092-8674(03)00714-1

14. Martin-Serrano J, Yaravoy A, Perez-Caballero D, Bieniasz PD. 2003. Divergent retroviral late-budding domains recruit vacuolar protein sorting factors by using alternative adaptor proteins. Proc Natl Acad Sci U S A 100:12414-12419. doi:10.1073/pnas.2133846100

15. Goff A, Ehrlich LS, Cohen SN, Carter CA. 2003. Tsg101 Control of Human Immunodeficiency Virus Type 1 Gag Trafficking and Release. J Virol 77:9173-9182. doi:10.1128/jvi.77.17.9173-9182.2003

16. Welker R, Kottler H, Kalbitzer HR, Kräusslich HG. 1996. Human immunodeficiency virus type 1 Nef protein is incorporated into virus particles and specifically cleaved by the viral proteinase. Virology 219:228-236. doi:10.1006/viro.1996.0240

17. Jenkins Y, Pornillos O, Rich RL, Myszka DG, Sundquist WI, Malim MH. 2001. Biochemical Analyses of the Interactions between Human Immunodeficiency Virus Type $1 \mathrm{Vpr}$ and p6Gag. J Virol 75:10537-10542. doi:10.1128/jvi.75.21.10537-10542.2001 
18. Kondo E, Göttlinger HG. 1996. A conserved LXXLF sequence is the major determinant in p6gag required for the incorporation of human immunodeficiency virus type $1 \mathrm{Vpr}$. $J$ Virol 70:159-64. Available at:

http://www.ncbi.nlm.nih.gov/pubmed/8523520\%0Ahttp://www.pubmedcentral.nih.gov/artic lerender.fcgi?artid=PMC189800

19. Muller B, Tessmer U, Schubert U, Krausslich H-G. 2000. Human Immunodeficiency Virus Type 1 Vpr Protein Is Incorporated into the Virion in Significantly Smaller Amounts than Gag and Is Phosphorylated in Infected Cells. J Virol 74:9727-9731. doi:10.1128/jvi.74.20.9727-9731.2000

20. Cohen E, Terwilliger E, Jalinoos Y, Proulx J, Sodroski J, Haseltine W. 1990. Identification of HIV-1 vpr product and function. J Acquir Immune Defic Syndr 1:

21. Lu YL, Spearman P, Ratner L. 1993. Human immunodeficiency virus type 1 viral protein $\mathrm{R}$ localization in infected cells and virions. J Virol 67:6542-6550.

22. Paxton W, Connor RI, Landau NR. 1993. Incorporation of Vpr into human immunodeficiency virus type 1 virions: requirement for the $\mathrm{p} 6$ region of gag and mutational analysis. J Virol 67:7229-37. Available at: http://www.ncbi.nlm.nih.gov/pubmed/8230445\%0Ahttp://www.pubmedcentral.nih.gov/artic lerender.fcgi?artid=PMC238185

23. Gibbs JS, Lackner AA, Lang SM, Simon MA, Sehgal PK, Daniel MD, Desrosiers RC. 1995. Progression to AIDS in the absence of a gene for vpr or vpx. J Virol 69:2378-83. Available at: http://www.ncbi.nlm.nih.gov/pubmed/7884883\%0Ahttp://www.pubmedcentral.nih.gov/artic lerender.fcgi?artid=PMC188910

24. Hirsch VM, Sharkey ME, Brown CR, Brichacek B, Goedstein S, Wakefield J, Byrum R, Elkins WR, Hahn BH, Lifson JD, et al. 1998. Vpx is required for dissemination and pathogenesis of SIV(SM) PBj: Evidence of macrophage-dependent viral amplification. Nat Med 4:1401-1408. doi:10.1038/3992

25. Gallay P, Stitt V, Trono D, Mundy C, Oettinger M. 1996. Role of the karyopherin pathway in human immunodeficiency virus type 1 nuclear import. J Virol 70:1027-1032.

26. Heinzinger NK, Bukrinsky MI, Haggerty SA, Ragland AM, Kewalramani V, Lee MA, Gendelman HE, Ratner L, Stevenson M, Emerman M. 1994. The Vpr protein of human immunodeficiency virus type 1 influences nuclear localization of viral nucleic acids in nondividing host cells. Proc Natl Acad Sci U S A 91:7311-7315. doi:10.1073/pnas.91.15.7311

27. Hrimech M, Yao XJ, Bachand F, Rougeau N, Cohen EA. 1999. Human immunodeficiency virus type 1 (HIV-1) Vpr functions as an immediate-early protein during HIV-1 infection. $J$ Virol 73:4101-9. Available at:

http://www.ncbi.nlm.nih.gov/pubmed/10196306\%0Ahttp://www.pubmedcentral.nih.gov/arti clerender.fcgi?artid=PMC104189

28. Poon B, Grovit-Ferbas K, Stewart SA, Chen ISY. 1998. Cell cycle arrest by Vpr in HIV-1 virions and insensitivity to antiretroviral agents. Science (80- ) 281:266-269. doi:10.1126/science.281.5374.266

29. Chang F, Re F, Sebastian S, Sazer S, Luban J. 2004. HIV-1 Vpr Induces Defects in Mitosis, Cytokinesis, Nuclear Structure, and Centrosomes. Mol Biol Cell 15:1793-1801. 
Available at: https://www.molbiolcell.org/doi/pdf/10.1091/mbc.e03-09-0691

30. Liu H, Wu X, Xiao H, Conway JA, Kappes JC. 1997. Incorporation of functional human immunodeficiency virus type 1 integrase into virions independent of the Gag-Pol precursor protein. $J$ Virol 71:7704-10. Available at:

http://www.pubmedcentral.nih.gov/articlerender.fcgi?artid=192121\&tool=pmcentrez\&rend ertype $=$ abstract

31. Wu X, Liu H, Xiao H, Conway JA, Hunter E, Kappes JC. 1997. Functional RT and IN incorporated into HIV-1 particles independently of the Gag/Pol precursor protein. EMBO J 16:5113-5122. doi:10.1093/emboj/16.16.5113

32. Xiaoyun WU, Liu H, Xiao H, Conway JA, Kappes JC. 1996. Inhibition of human and simian immunodeficiency virus protease function by targeting $\mathrm{Vpx}$-protease-mutant fusion protein into viral particles. J Virol 70:3378-3384.

33. Fletcher TM, Soares MA, McPhearson S, Hui H, Wiskerchen MA, Muesing MA, Shaw GM, Leavitt AD, Boeke JD, Hahn BH. 1997. Complementation of integrase function in HIV-1 virions. EMBO J 16:5123-5138. doi:10.1093/emboj/16.16.5123

34. Bouyac-Bertoia M, Dvorin JD, Fouchier RAM, Jenkins Y, Meyer BE, Wu LI, Emerman M, Malim MH. 2001. HIV-1 Infection Requires a Functional Integrase NLS. Mol Cell 7:10251035. doi:10.1016/S1097-2765(01)00240-4

35. Muthumani K, Montaner LJ, Ayyavoo V, Weiner DB. 2004. Vpr-GFP Virion Particle Identifies HIV-Infected Targets and Preserves HIV-1Vpr Function in Macrophages and TCells. DNA Cell Biol 19: doi:https://www.liebertpub.com/doi/pdf/10.1089/104454900314564

36. Desai TM, Marin M, Sood C, Shi J, Nawaz F, Aiken C, Melikyan GB. 2015. Fluorescent protein-tagged Vpr dissociates from HIV-1 core after viral fusion and rapidly enters the cell nucleus. Retrovirology 12:1-20. doi:10.1186/s12977-015-0215-Z

37. Campbell EM, Perez O, Melar M, Hope TJ. 2007. Labeling HIV-1 virions with two fluorescent proteins allows identification of virions that have productively entered the target cell. Virology 360:286-293. doi:10.1016/j.virol.2006.10.025

38. Votteler J, Ogohara C, Yi S, Hsia Y, Nattermann U, Belnap DM, King NP, Sundquist WI. 2016. Designed proteins induce the formation of nanocage-containing extracellular vesicles. Nature 540:292-295. doi:10.1038/nature20607

39. Jones DM, Padilla-Parra S. 2015. Imaging real-time HIV-1 virion fusion with FRET-based biosensors. Sci Rep 5:1-10. doi:10.1038/srep13449

40. Larson DR, Johnson MC, Webb WW, Vogt VM. 2005. Visualization of retrovirus budding with correlated light and electron microscopy. Proc Natl Acad Sci U S A 102:1545315458. doi:10.1073/pnas.0504812102

41. Chutiwitoonchai N, Siarot L, Takeda E, Shioda T, Ueda M, Aida Y. 2016. HIV-1 Vpr abrogates the effect of TSG101 overexpression to support virus release. PLoS One 11:117. doi:10.1371/journal.pone. 0163100

42. Khan MA, Aberham C, Kao S, Akari H, Gorelick R, Bour S, Strebel K. 2001. Human Immunodeficiency Virus Type 1 Vif Protein Is Packaged into the Nucleoprotein Complex through an Interaction with Viral Genomic RNA. J Virol 75:7252-7265. 
43. Bentham M, Mazaleyrat S, Harris M. 2006. Role of myristoylation and N-terminal basic residues in membrane association of the human immunodeficiency virus type 1 Nef protein. J Gen Virol 87:563-571. doi:10.1099/vir.0.81200-0

44. Pandori MW, Fitch NJ, Craig HM, Richman DD, Spina CA, Guatelli JC. 1996. Producercell modification of human immunodeficiency virus type 1: Nef is a virion protein. $J$ Virol 70:4283-90. Available at:

http://www.ncbi.nlm.nih.gov/pubmed/8676450\%0Ahttp://www.pubmedcentral.nih.gov/artic lerender.fcgi?artid=PMC190360

45. Welker R, Cardel B, Kräusslich HG, Harris M. 1998. Virion incorporation of human immunodeficiency virus type $1 \mathrm{Nef}$ is mediated by a bipartite membrane-targeting signal: Analysis of its role in enhancement of viral infectivity. $J$ Virol 72:8833-8840.

46. Bukovsky AA, Dorfman T, Weimann A, Göttlinger HG. 1997. Nef association with human immunodeficiency virus type 1 virions and cleavage by the viral protease. $J$ Virol 71:1013-8. Available at: http://www.pubmedcentral.nih.gov/articlerender.fcgi?artid=191151\&tool=pmcentrez\&rend ertype $=$ abstract

47. Toohey K, Wehrly K, Nishio J, Perryman S, Chesebro B. 1995. Human immunodeficiency virus envelope $\mathrm{V} 1$ and $\mathrm{V} 2$ regions influence replication efficiency in macrophages by affecting virus spread. Virology 213:70-79. doi:10.1006/viro.1995.1547

48. Wehrly K, Chesebro B. 1997. P24 Antigen Capture Assay for Quantification of Human Immunodeficiency Virus Using Readily Available Inexpensive Reagents. Methods A Companion to Methods Enzymol 12:288-293. doi:10.1006/meth.1997.0481 